\title{
On some particular regular Diophantine 3-tuples
}

\author{
Ö. Özer ${ }^{a, *}$, Z. C. Sahin ${ }^{\mathrm{b}}$ \\ ${ }^{a}$ Department of Mathematics, Faculty of Science and Arts, Kirklareli University, Kirklareli, 39100, Turkey. \\ ${ }^{b}$ Department of Mathematics, Faculty of Science and Arts, Sŭleyman Demirel University, Isparta, Turkey.
}

\begin{abstract}
Diophantine $n$-tuple where $n=3$ is called as a Diophantine triple. It means that Diophantine triple is a set of three positive integers satisfying special condition. For example, $\{a, b, c\}$ is called a $D(k)$-Diophantine triple if multiplying of any two different of them plus $\mathrm{k}$ is a perfect square integer where $\mathrm{k}$ is an integer.

In this work, we take in consideration some kind of regular $\mathrm{D}\left( \pm 3^{3}\right)$-Diophantine triples. We demonstrate that such sets can not be extendible to $\mathrm{D}\left( \pm 3^{3}\right)$-Diophantine quadruple by using algebraic methods such as classical Pell equations solutions, solutions of $u x^{2}+v y^{2}=w$ Diophantine equations where $u, v, w \in \mathbb{Z}$, factorization in the set of integers, and so on. Besides, we obtain some notable characteristic properties for such sets.
\end{abstract}

Keywords: Diophantine Triple, Pell equations, Diophantine equations, modular arithmetic, reciprocity theorem, Legendre symbol.

2010 MSC: 11Dxx, 11C08, 11G99.

(C)2018 All rights reserved.

\section{Introduction}

Number theory is one of the most significant fields of mathematics, especially, primes, prime factorization and diophantine equations have a great importance in number theory. A set of $m$ distinct positive integers $\left\{\delta_{1}, \delta_{2}, \ldots, \delta_{m}\right\}$ is called a Diophantine m-tuple with $k$ and represented as $D(k)$ or $P_{k}$ if $\delta_{i} \delta_{j}+k(i \neq j, i, j=1,2, m)$ is a perfect square integer. Although the topic of Diophantine $m$-tuple is very ancient problem, still many authors have been working on it with different techniques.

Bashmakova [2] gave the definition of Diophantine m-tuple as the statement: If we choose $k=1$ in the above mentioned definition (Diophantine $\mathrm{m}$-tuple with $\mathrm{k}$ ), we get the set of $\mathrm{m}$ positive integers which is called a Diophantine m-tuple while the product of any two of its distinct elements increased by 1 is a perfect square integer.

Baker and Davenport [1] considered the general solutions of each separated equations $3 x^{2}-2=y^{2}$ and $8 x^{2}-7=z^{2}$ by using algebraic number theory. Brown [4] proved some general results for $P_{k}$ while $k=2(\bmod 4)$ and demonstrated that the $P_{(-1)}$ set $\{1,2,5\}$ can not be extendable. The book [6]

\footnotetext{
*Corresponding author

Email address: ozenozer39@gmail.com (Ö. Özer)
}

doi: $10.22436 / \mathrm{mns} .03 .01 .04$

Received: 2018-11-14 Revised: 2019-01-03 Accepted: 2019-01-30 
written by Dickson, has many crucial results on number theory and group theory. Deshpande [5] created Diophantine triples considering a recurrence relation in the terms of a special sequence. Dujella and Jurasic [7] defined the some types of regular Diophantine m-tuples and obtained significant results for them.

Fermat [8] worked on the problem over integers considering the quadruple set $\{1,3,8,120\}$. Gopalan et al. ([12-14]) constructed different types of interesting triple sets by creating new Dio triple set definition. Also, the author Gopalan and Özer [15] prepared a book on the varied types of Pell equations. Kedlaya [16] determined a new elementary method to solve special systems of Diophantine equation Özer [19, 22] proved some varied types of Diophantine triples using different algebraic methods.

Readers can get many significant and valuable information on number theory in the lecture notes of Goldmakher [11], Kurur and Saptharishi [17], and the books of Mollin [18] and Roberts [23]. Besides, one may refer $[3,9,10]$ for an extensive review of various problems on Diophantine m-tuples.

In this paper, we consider several types of $\mathrm{D}\left( \pm 3^{3}\right)$-Diophantine triples. Firstly, we demonstrate that they are regular Diophantine triples. Secondly, we prove that they can not extendable to $D\left( \pm 3^{3}\right)$ Diophantine quadruples. Lastly, we give some results on the characterization of the elements of $D\left( \pm 3^{3}\right)$ Diophantine triples using algebraic structures in algebraic and elementary number theory.

\section{Preliminaries}

Definition 2.1 ([11, Quadratic residue]). Let $\mathrm{p}$ be an odd prime, $\alpha \equiv 0(\bmod p)$. We say that $\alpha$ is a quadratic residue $\bmod p$ if $\alpha$ is a square $\bmod p$ (it is a quadratic non-residue otherwise).

Lemma $2.2([11])$. Let $\alpha \equiv 0(\bmod p)$. Then $\alpha$ is a quadratic residue $\bmod p$ if and only if $\alpha^{(p-1) / 2} \equiv 1(\bmod p)$.

Definition 2.3 ([11, Legendre symbol]). Legendre symbol introduces the following notation for prime $p$ :

$$
(\alpha / p)= \begin{cases}0, & \text { if } p / \alpha, \\ 1, & \text { if } x^{2}=a(\bmod p) \text { has a nonzero solution, } \\ -1, & \text { if } x^{2} \equiv a(\bmod p) \text { has no solution. }\end{cases}
$$

Lemma 2.4. Let $(\vdots)$ be Legendre symbol and $\mathrm{p}$ is prime. Then, followings are satisfied.

$$
\begin{aligned}
\left(\frac{-1}{p}\right) & =\left\{\begin{array}{ll}
1, & \text { if } p \equiv 1(\bmod 4), \\
-1, & \text { if } p \equiv-1(\bmod 4),
\end{array},\right. \\
\left(\frac{3}{p}\right) & = \begin{cases}1, & \text { if } p \equiv \pm 1(\bmod 12), \\
-1, & \text { if } p \equiv \pm 5(\bmod 12),\end{cases} \\
\left(\frac{2}{p}\right) & = \begin{cases}1, & \text { if } p \equiv \pm 1(\bmod 8), \\
-1, & \text { if } p \equiv \pm 3(\bmod 8),\end{cases} \\
\left(\frac{5}{p}\right) & = \begin{cases}1, & \text { if } p \equiv \pm 1(\bmod 5), \\
-1, & \text { if } p \equiv \pm 2(\bmod 5) .\end{cases}
\end{aligned}
$$

Definition 2.5 ([11, Jacobi symbol]). It is convenient to extend the Legendre symbol $\left(\frac{\alpha}{p}\right)$ to a symbol $\left(\frac{\alpha}{m}\right)$, where $m$ is an arbitrary odd integer; this generalization is called the Jacobi symbol. Whenever $m$ is an odd prime, we take $\left(\frac{\alpha}{m}\right)$ to be the Legendre symbol. We now extend this by multiplicativity to all positive odd integers $m$, i.e., if $m=p_{1}^{s_{1}} \cdots p_{k}^{s_{k}}$ where the $p_{i}$ are odd primes, set

$$
\left.\left(\frac{\alpha}{m}\right)=\left(\frac{\alpha}{p_{1}}\right)^{s_{1}} \cdots \frac{\alpha}{p_{k}}\right)^{s_{k}}
$$

As usual with empty products, we set $\left(\frac{\alpha}{1}\right)=1$. 
Theorem 2.6 ([17, Reciprocity theorem $])$. If $\mathrm{p} \neq \mathrm{q}$ are odd primes, then

$$
\left(\frac{p}{q}\right)\left(\frac{p}{q}\right)=(-1)^{\frac{p-1}{2} \frac{q-1}{2}}= \begin{cases}1, & \text { if } p \text { or } q \equiv \pm 1(\bmod 4), \\ -1, & \text { otherwise. }\end{cases}
$$

Theorem 2.7 ([17, Quadratic reciprocity law]). If $\mathrm{m}, \mathrm{n}$ are odd numbers such that $(\mathrm{m}, \mathrm{n})=1$, then

$$
\begin{aligned}
\left(\frac{2}{n}\right) & =(-1)^{\frac{n^{2}-1}{8}}, \\
\left(\frac{m}{n}\right)\left(\frac{n}{m}\right) & =(-1)^{\frac{(m-1)}{2} \frac{(n-1)}{2} .}
\end{aligned}
$$

Definition $2.8([7])$. A $D(m)$-triple $\{u, v, w\}$ is called regular if it satisfies the condition

$$
(w-v-u)^{2}=4(u \cdot v+m) .
$$

\section{Theorems and results}

Theorem 3.1. A set $\mathrm{D}\left(3^{3}\right)=\{1,9,22\}$ is regular Diophantine triple but can not be extended to $\mathrm{D}\left(3^{3}\right)$-Diophantine quadruple.

Proof. If we consider Definition 2.4 and apply (2.10) to $\{1,9,22\}$, it is seen that $\mathrm{D}\left(3^{3}\right)=\{1,9,22\}$ is a regular Diophantine set. We suppose that $\{1,9,22\}$ can be extended to quadruple for any positive integer $\beta$. Then, $\{1,9,22, \beta\}$ is a $D(27)$ Diophantine set. So, there exist $a, b, c$ integers such that;

$$
\begin{aligned}
\beta+27 & =a^{2}, \\
9 \beta+27 & =b^{2}, \\
22 \beta+27 & =c^{2} .
\end{aligned}
$$

By eliminating $\beta$ between (3.1) and (3.2), we have

$$
9 a^{2}-b^{2}=216
$$

In (3.4), the left side can be written as difference of two squares since 9 is a perfect square, so, we have $(3 a-b)(3 a+b)=216$. Factorizing 216 as finitely, we have Table 1 .

\begin{tabular}{|} 
Table 1: Solutions of $9 \mathrm{a}^{2}-\mathrm{b}^{2}=216$. \\
\begin{tabular}{|c|c|c|}
\hline Solution & Class 1 solutions 1 & Class 2 solutions \\
$\mathrm{a}$ & \pm 7 & \pm 5 \\
$\mathrm{~b}$ & \pm 15 & \pm 3 \\
\hline
\end{tabular}
\end{tabular}

By dropping $\beta$ between (3.1) and (3.3), then we have

$$
22 a^{2}-c^{2}=567
$$

Considering solution 1 , substituting $a^{2}=49$ into (3.5) we get $c^{2}=511$ where $c$ is not an integer solution. In the same way, substituting another solution $a^{2}=25$ into the (3.5), we obtain $c^{2}=-17$ which is a contradiction. It is shown that any value of $c$ is not integer for the solution of (3.5). So, there is no such $\beta \in \mathbb{Z}^{+}$and the set $P_{+27}=\{1,9,22\}$ can not be extended.

Theorem 3.2. $A \mathrm{P}_{+27}=\{1,142,169\}$ set is both regular and non-extendible to $\mathrm{P}_{+27}$-Diophantine quadruple. 
Proof. We can easily see that $\{1,142,169\}$ is a regular $\mathrm{P}_{+27}$ triple set from (2.8) in the Definition 2.8. Suppose that there exists a positive integer $d$ such that $\{1,142,169, d\}$ is a $P_{+27}$ set. Then the following equations have integral solutions $x, y, z$ in the set of integers.

$$
\begin{aligned}
d+27 & =u^{2}, \\
142 d+27 & =v^{2}, \\
169 d+27 & =w^{2} .
\end{aligned}
$$

From (3.6) and (3.8), we obtain

$$
169 u^{2}-w^{2}=4536
$$

By factorizing the left side of (3.9), we have

$$
(13 u-w)(13 u+w)=4536
$$

If we search the solutions of the (3.10), we get Table 2 .

Table 2: Solutions of $169 u^{2}-w^{2}=4536$.

\begin{tabular}{|c|c|c|}
\hline $\begin{array}{c}\text { Solution } \\
(\mathrm{u}, \mathrm{w})\end{array}$ & $\begin{array}{c}\text { Class 1 solutions } \\
( \pm 15, \pm 183)\end{array}$ & $\begin{array}{c}\text { Class 2 solutions } \\
( \pm 13, \pm 155)\end{array}$ \\
\hline
\end{tabular}

From (3.6) and (3.7), we have

$$
142 u^{2}-v^{2}=3807
$$

If we substituting $u^{2}=225$ or $u^{2}=169$ into the (3.11), we obtain $v^{2}=28143$ or $v^{2}=20191$ which they are not integer solutions of (3.11) in return. Hence, $P_{+27}=\{1,142,169\}$ is non-extendible to $P_{+27}$-Diophantine quadruple.

Theorem 3.3. $A \mathrm{P}_{+27}=\{1,169,198\}$ set is not only regular triple but also non-extendible.

Proof. Let consider the set $\{1,169,198\}$. By applying (2.8) condition into the elements of the set, it is seen that $P_{+27}=\{1,169,198\}$ is a regular Diophantine triple. Assume that there exists a positive integer $V$ such that $\{1,169,198, \mathcal{V}\}$ is a $P_{27}$ quadruple. Then there are $x, y, z$ integers such that

$$
\begin{aligned}
\mathcal{V}+27 & =x^{2}, \\
169 \mathcal{V}+27 & =y^{2}, \\
198 \mathcal{V}+27 & =z^{2} .
\end{aligned}
$$

By dropping $\mathcal{V}$ between (3.12) and (3.13), we obtain

$$
169 x^{2}-y^{2}=4536
$$

and from (3.12) and (3.14) we get

$$
198 x^{2}-z^{2}=5319
$$

In (3.15), if we use factorization of integers, we write left side as $(13 x-y)(13 x+y)=4536$. Also, 4536 can be factorized as finitely. So, integer solutions of (3.15) are as Table 2 for $(x, y)$. If we substitute $x^{2}=225$ or $x^{2}=169$ into the (3.16), we have $z^{2}=39231$ or $z^{2}=28143$, where they are not integer solutions of (3.16), respectively.

Thus, there is no such $\mathcal{V} \in \mathbb{Z}^{+}$and the $\{1,169,198\}$ cannot be extended to $P_{27}$ quadruple. 
Remark 3.4. There are many different types of regular $P_{+27}$-Diophantine triples such as $\{2,11,27\},\{2,47,71\}$, $\{3,18,39\},\{3,66,99\},\{6,9,33\},\{9,13,46\},\{11,18,59\}$, and so on. In here, we just prove some of them by applying factorization method in the set of integers.

Theorem 3.5. There isn't any set $\mathrm{P}_{+27}$ including elements divided by $4,5,7$, or 17 .

Proof.

(a) We suppose that $u$ is an element of set $P_{+27}$. If $4 \alpha$ is also an element of set $P_{+27}$ for $\alpha \in \mathbb{Z}$, then we have

$$
4 \alpha u+27=A^{2}
$$

must be satisfied for some integer A. Applying ( $\bmod 4)$ on the both sides of (3.17), we obtain following inequality

$$
A^{2} \equiv 3(\bmod 4)
$$

If $A$ is odd integer then we get $A^{2} \equiv 1(\bmod 4)$ and also $A^{2} \equiv 0(\bmod 4)$ for even integer $A$. So, (3.18) can not solvability. This is a contradiction. Therefore, $4 \alpha$ can not be an element of $P_{+27}$ for any $\alpha \in \mathbb{Z}$.

(b) We suppose that $v$ and $5 \beta,(\beta \in Z)$ are elements of the set $P_{+27}$, then

$$
5 \beta v+27=B^{2}
$$

is satisfied for integer B. Applying (mod 5) to (3.19), we get

$$
\mathrm{B}^{2} \equiv 2(\bmod 5)
$$

has solutions if and only if Legendre symbol is $\left(\frac{2}{5}\right)=1$. If we consider Lemma 2.4 and applying (2.4), we obtain $\left(\frac{2}{5}\right)=-1$ which implies that (3.20) has no solution. This is a contradiction. Consequently, there is no set $\mathrm{P}_{+27}$ involving elements with 5 .

(c) In a similar way of (a) or (b), assume that $w$ is an element of set $P_{+27}$. If $7 \gamma,(\gamma \in \mathbb{Z})$ is also an element of set $\mathrm{P}_{+27}$, then

$$
7 \gamma w+27=c^{2}
$$

is obtained for integer $C$. If we apply $(\bmod 7)$, we have

$$
C^{2} \equiv-1(\bmod 7)
$$

Using (2.2) from Lemma 2.4, we have

$$
\left(\frac{-1}{7}\right)=(-1)^{\frac{7-1}{2}}=-1 \text {. }
$$

This shows that equation (3.21) is unsolvable. Hence, $7 \gamma$ can not be an element of $P_{+27}$ for $\gamma \in \mathbb{Z}$.

(d) In the same manner, suppose that $r$ and $17 \theta,(\theta \in \mathbb{Z})$ are elements of set $P_{+27}$. Then

$$
17 \theta \mathrm{r}+27=\mathrm{D}^{2}
$$

has to get solution for integer D. Applying $(\bmod 17)$ to (3.22), we get

$$
\mathrm{D}^{2} \equiv 10(\bmod 17)
$$

Using (2.7) of Theorem 2.7, then we obtain

$$
\left(\frac{10}{17}\right)=\left(\frac{2}{17}\right)\left(\frac{5}{17}\right)
$$

Applying (2.4) and (2.5) of Lemma 2.4 into the (3.24), we have $\left(\frac{2}{17}\right)=+1$ and $\left(\frac{5}{17}\right)=-1$. These imply that $\left(\frac{10}{17}\right)=-1$ and the equation (3.23) isnt solvable. Therefore, $17 \theta$ can not be an element of $P_{+27}$ for $\theta \in \mathbb{Z}$. 
Theorem 3.6. $A \mathrm{D}(-27)=\{1,31,36\}$ set can not be extended to $\mathrm{D}(-27)$ quadruple but it is regular $\mathrm{D}(-27)$ Diophantine triple.

Proof. The set $\{1,31,36\}$ has the property of $\mathrm{D}(-27)$ Diophantine set. From (2.8) of Definition 2.8, it is clear that $\{1,31,36\}$ is a regular $\mathrm{D}(-27)$ Diophantine triple. Let show that the set is non-extendible. Let $\mu$ be any other positive integer in $\{1,31,36, \mu\}$. Then following equations hold for some $X, Y, Z$ integers.

$$
\begin{aligned}
\mu-27 & =X^{2}, \\
31 \mu-27 & =Y^{2}, \\
36 \mu-27 & =Z^{2} .
\end{aligned}
$$

Elimination of $\mu$ between (3.25) and (3.27) as well as between (3.25) and (3.26), we obtain following equations, respectively.

$$
\begin{aligned}
Z^{2}-36^{2} & =945 \\
Y^{2}-31 X^{2} & =810 .
\end{aligned}
$$

By using factorization, solutions of (3.28) are obtained as Table 3. Putting solutions from Table 3 into the

Table 3: Solutions of $Z^{2}-36 X^{2}=945$.

\begin{tabular}{|c|c|c|c|c|}
\hline $\begin{array}{c}\text { Solutions } \\
(\mathrm{X}, \mathrm{Z})\end{array}$ & $\begin{array}{c}\text { Class 1 solutions } \\
( \pm 26, \pm 159)\end{array}$ & $\begin{array}{c}\text { Class 1 solutions } \\
( \pm 8, \pm 57)\end{array}$ & $\begin{array}{c}\text { Class 2 solutions } \\
( \pm 4, \pm 39)\end{array}$ & $\begin{array}{c}\text { Class 3 solutions } \\
( \pm 2, \pm 33)\end{array}$ \\
\hline
\end{tabular}

(3.29), we have $Y^{2}=21766, Y^{2}=2794, Y^{2}=1306$ or $Y^{2}=934$ for $X^{2}=676, X^{2}=64, X^{2}=16$ or $X^{2}=4$, respectively. These imply that they (values of $Y$ ) are not integer solution of (3.29). Thus, there is no such $\mu \in \mathbb{Z}^{+}$and the $\mathrm{D}(-27)=\{1,31,36\}$ set is non-extendible to Diophantine $\mathrm{D}\left(-3^{3}\right)$ quadruple.

Theorem 3.7. $A \mathrm{D}\left(-3^{3}\right)=\{1,36,43\}$ is regular triple set however it can not be extended.

Proof. Diophantine triple $\mathrm{D}\left(-3^{3}\right)=\{1,36,43\}$ is regular triple set since it satisfies (2.8) condition of Definition 2.8. Assume that $\{1,36,43, \varphi\}$ is a $D\left(-3^{3}\right)$ Diophantine quadruple. So, there are $A, B, C$ integers such that

$$
\begin{aligned}
\varphi-27 & =A^{2}, \\
36 \varphi-27 & =B^{2}, \\
43 \varphi-27 & =C^{2} .
\end{aligned}
$$

Dropping $\varphi$ between (3.30) and (3.31), we obtain

$$
B^{2}-36 A^{2}=945
$$

and similarly from (3.30) and (3.32), we have

$$
C^{2}-43 A^{2}=1134
$$

The Table 3 gives the solutions of (3.33) if we take $(A, B)$ instead of $(X, Z)$ in such table. Putting values of $A^{2}$ from Table 3 into the (3.34), respectively, we obtain $C^{2}=30202, C^{2}=3886, C^{2}=1822$, or $C^{2}=1306$. It shows that $C$ is not integer yields (3.34). So, there is no positive $\varphi$ integer and the $D\left(-3^{3}\right)=\{1,36,43\}$ is not extendible to $\mathrm{D}\left(-3^{3}\right)$ Diophantine quadruple.

Theorem 3.8. A Diophantine triple $\mathrm{P}_{\left(-3^{3}\right)}=\{2,18,26\}$ is regular and non-extendible to $\mathrm{D}\left(-3^{3}\right)$ Diophantine quadruple. 
Proof. From (2.8) in Definition 2.8, it is clear that $\{2,18,26\}$ is regular Diophantine triple. Assume that there exists a positive integer $k$ such that $\{2,18,26, k\}$ is a $P_{\left(-3^{3}\right)}$ quadruple. Then there exist $x, y, z$ integers such that

$$
\begin{aligned}
2 k-27 & =x^{2}, \\
18 k-27 & =y^{2}, \\
26 k-27 & =z^{2} .
\end{aligned}
$$

Dropping $k$ from (3.35) and (3.36), we obtain $y^{2}-9 x^{2}=216$. Using factorization, we have the solutions of $y^{2}-9 x^{2}=216$ as Table 4 .

Table 4: Solutions of $y^{2}-9 x^{2}=216$.

\begin{tabular}{|c|c|c|}
\hline $\begin{array}{c}\text { Solutions } \\
(\mathrm{x}, \mathrm{y})\end{array}$ & $\begin{array}{c}\text { Class 1 solutions } \\
( \pm 5, \pm 21)\end{array}$ & $\begin{array}{c}\text { Class } 2 \text { solutions } \\
( \pm 1, \pm 15)\end{array}$ \\
\hline
\end{tabular}

From (3.35) and (3.37), we get

$$
z^{2}-13 x^{2}=324
$$

Substituting solutions $x^{2}=25$ or $x^{2}=1$ into the (3.38), we have $z^{2}=649$ or $z^{2}=337$ which are not integer solutions of (3.38), respectively. Thus, there is no such $k \in \mathbb{Z}^{+}$and the $P_{-3^{3}}=\{2,18,26\}$ set can not be extended to $\mathrm{P}_{-3^{3}}$ Diophantine quadruple.

Theorem 3.9. $A \mathrm{P}_{-27}=\{3,9,12\}$ set can not be extended to $\mathrm{P}_{-3^{3}}$ Diophantine quadruple however it is regular $\mathrm{P}_{-27}$-Diophantine triple.

Proof. It is trivial that $\{3,9,12\}$ triple is regular from (2.8). Let us determine whether or not there is any other positive integer in this set. Assume that $\{3,9,12, d\}$ is $P_{-27}$ Diophantine quadruple. Then following equations are satisfied for some $a, b, c$ integers.

$$
\begin{array}{r}
3 d-27=a^{2}, \\
9 d-27=b^{2}, \\
12 d-27=c^{2}
\end{array}
$$

Elimination of $d$ between (3.39) and (3.41) as well as (3.39) and (3.40), we have

$$
\begin{aligned}
& c^{2}-4 a^{2}=81, \\
& b^{2}-3 a^{2}=54 .
\end{aligned}
$$

Solutions of (3.42) can be given as Table 5.

Table 5: Solutions of $c^{2}-4 a^{2}=81$.

\begin{tabular}{|c|c|c|c|}
\hline $\begin{array}{c}\text { Solutions } \\
(a, c)\end{array}$ & $\begin{array}{c}\text { Class 1 solutions } \\
( \pm 20, \pm 41)\end{array}$ & $\begin{array}{c}\text { Class 2 solutions } \\
( \pm 6, \pm 15)\end{array}$ & Class 3 solutions \\
$( \pm 0, \pm 9)$ \\
\hline
\end{tabular}

Putting solutions into the (3.43), we get $b^{2}=1254, b^{2}=162$ or $b^{2}=54$ which they are not integer solution of (3.43). Thus, there is no such $d \in Z^{+}$and the $P_{-27}=\{3,9,12\}$ Diophantine triple isnt extendible to $\mathrm{P}_{-27}$ quadruple.

Theorem 3.10. Sets $\mathrm{P}_{-27}=\{4,7,9\}$ and $\mathrm{P}_{-27}=\{4,9,19\}$ are regular Diophantine triple but can not be extended to $\mathrm{P}_{-27}$-Diophantine quadruple. 
Proof. Both of the $\mathrm{P}_{-27}$ sets in the Theorem 3.10 are regular since (2.8) condition is satisfied. Assume that $\{4,7,9, \alpha\}$ is a $P_{-27}$ set. There are $X, Y, Z$ integers such that,

$$
\begin{aligned}
& 4 \alpha-27=X^{2}, \\
& 7 \alpha-27=Y^{2}, \\
& 9 \alpha-27=Z^{2} .
\end{aligned}
$$

Eliminating $\alpha$ between (3.44) and (3.46), we obtain

$$
-9 X^{2}+4 Z^{2}=135
$$

and similarly, dropping $\alpha$ between (3.44) and (3.45) we have

$$
-7 X^{2}+4 Y^{2}=81
$$

By (3.47), we have $(2 Z-3 X)(2 Z+3 X)=135$ and solutions are found as $(X, Z)=( \pm 7, \pm 12)$ or $(X, Z)=$ $( \pm 1, \pm 6)$. Substituting solutions $X^{2}=49$ or $X^{2}=1$ into the (3.48), we get $Y^{2}=106$ or $Y^{2}=22$ in return. It proves that $Y$ is not integer yields (3.48). So, there is no $\alpha$ integer and the $P_{-27}=\{4,7,9\}$ is non-extendible to quadruple. In a similar way, there are $x, y, z$ integers such that

$$
\begin{gathered}
4 \beta-27=x^{2}, \\
9 \beta-27=y^{2}, \\
19 \beta-27=z^{2}
\end{gathered}
$$

for $P_{-27}=\{4,9,19, \beta\}$ Diophantine quadruple. Dropping $\beta$ between (3.49) and (3.50) we get the following equation same as solutions of (3.47) are obtained,

$$
(x, y)=( \pm 7, \pm 12), \quad(x, y)=( \pm 1, \pm 6) .
$$

Eliminating $\beta$ between (3.49) and (3.51), we get $4 z^{2}-19 x^{2}=405$. Substituting solutions $(x, y)=( \pm 7, \pm 12)$, $(x, y)=( \pm 1, \pm 6)$ into the $4 z^{2}-19 x^{2}=405$ then we have $z^{2}=334$ or $z^{2}=106$ such that $z$ is not integer. Hence, there is no such positive $\beta$ integer and the $P_{-27}=\{4,9,19\}$ set is non-extendible to Diophantine $P_{-27}$ quadruple.

Theorem 3.11. There isn't any set $\mathrm{P}_{-27}$ including $\mathrm{R}$ elements satisfying any of following conditions:

(i) $\mathrm{R} \in \mathbb{Z}, \mathrm{R} \equiv 0(\bmod 5)$;

(ii) $\mathrm{R} \in \mathbb{Z}, \mathrm{R} \equiv 0(\bmod 8)$;

(iii) $R \in \mathbb{Z}, R \equiv 0(\bmod 11)$;

(iv) $\mathrm{R} \in \mathbb{Z}, \mathrm{R} \equiv 0(\bmod 17)$.

Proof.

(i) If $R=5 u$ and $t$ are elements of set $P_{-27}$ for $u \in \mathbb{Z}$, then we obtain

$$
5 u t-27=X^{2} \text {. }
$$

Applying (mod 5) on both sides of the (3.52), we have

$$
X^{2} \equiv 3(\bmod 5)
$$

Using (2.6) of Theorem 2.6, we get

$$
\left(\frac{3}{5}\right)\left(\frac{5}{3}\right)=(-1)^{\frac{3-1}{2} \frac{5-1}{2}}=-1
$$

And $\left(\frac{5}{3}\right)=\left(\frac{2}{3}\right)=-1$ from (2.4) in Lemma 2.4. So, we obtain

$$
\left(\frac{3}{5}\right)=-1
$$

This is a contradiction. So, there is no $P_{-27}$ set containing any elements such that $R \in \mathbb{Z}, R \equiv 0(\bmod 5)$. 
(ii) Assume that $s$ and $8 v,(v \in \mathbb{Z})$ are elements of set $P_{-27}$, then we have

$$
8 v s-27=Y^{2}
$$

for integer $Y$. Also, $Y^{2} \equiv 5(\bmod 8)$ is satisfied. By Lemma 2.1 and considering residue classes $(\bmod 8)$, we get $Y^{2} \equiv 0,1,4(\bmod 8)$. So, 5 is non-quadratic residue $(\bmod 8)$ and it is a contradiction. Hence, there isnt any set $P_{-27}$ including any element satisfying $R \in \mathbb{Z}, R \equiv 0(\bmod 8)$.

(iii) If $m$ and $R \in \mathbb{Z}, R \equiv 0(\bmod 11)$ are elements of set $P_{-27}$,

$$
11 \mathrm{tm}-27=\mathrm{Z}^{2}
$$

satisfies for integer Z. Applying (modulo 11), we get

$$
Z^{2} \equiv 6(\bmod 11)
$$

Considering property of Legendre symbol from Definition 2.3, we have

$$
\left(\frac{6}{11}\right)=\left(\frac{2}{11}\right)\left(\frac{3}{11}\right)
$$

From (2.4) and (2.3), we obtain $\left(\frac{2}{11}\right)=-1$ and $\left(\frac{3}{11}\right)=+1$. So, we get $\left(\frac{6}{11}\right)=-1$. The equation (3.53) is not solvable. Thus, $R \in \mathbb{Z}, R \equiv 0(\bmod 11)$ can not be an element of $P_{-27}$.

(iv) Assume that $t$ is an element of set $P_{-27}$, if $R \in \mathbb{Z}, R \equiv 0(\bmod 17)$ is also an element of set $P_{-27}$, then

$$
17 \mathrm{tn}-27=\mathrm{T}^{2}
$$

for integer T. If we apply (mod 17) on the both sides of (3.54), we get

$$
\mathrm{T}^{2} \equiv 7(\bmod 17)
$$

Using Theorem 2.6, we have

$$
\left(\frac{7}{17}\right)\left(\frac{17}{7}\right)=(-1)^{\left(\frac{7-1}{2}\right)\left(\frac{17-1}{2}\right)}=1
$$

From the Legendre symbol's property, we can write $\left(\frac{17}{7}\right)=\left(\frac{3}{7}\right)$. Using $(2.3)$ in Lemma 2.4, we obtain that $\left(\frac{3}{7}\right)=-1$. So, we have

$$
\left(\frac{7}{17}\right)=-1
$$

This shows that there is no integer $T$ satisfying (3.54). It is a contradiction. Therefore, there is no set $\mathrm{P}_{-27}$ including any elements such that $R \in \mathbb{Z}, R \equiv 0(\bmod 17)$.

Remark 3.12. Using similar technique, readers also can prove that $\{1,36,91\},\{1,171,196\},\{3,12,21\},\{4,27,49\}$, $\{4,49,79\},\{6,18,42\},\{7,9,28\}$, and so on. They are regular $\mathrm{D}\left(-3^{3}\right)$ Diophantine triples although they aren't extendible $\mathrm{D}\left(-3^{3}\right)$ Diophantine quadruple.

\section{References}

[1] A. Baker, H. Davenport, The equations $3 x^{2}-2=y^{2}$ and $8 x^{2}-7=z^{2}$, Quart. J. Math. Oxford Ser. (2), 20 (1969), 129-137. 1

[2] I. G. Bashmakova, Diophantus of Alexandria: Arithmetics and The Book of Polygonal Numbers, Nauka, Moskow, (1974). 1

[3] A. F. Beardon, M. N. Deshpande, Diophantine triples, The Mathematical Gazette, 86 (2002), 258-260. 1

[4] E. Brown, Sets in which $x y+k$ is Always a Square, Math. Comp., 45 (1985), 613-620. 1 
[5] M. N. Deshpande, On interesting family of Diophantine triples, Int. J. Math. Ed. Sci. Tech., 33 (2002), 253-256. 1

[6] L. E. Dickson, History of Theory of Numbers: Diophantine Analysis, Dove Publ., New York, (2005). 1

[7] A. Dujella, A. Jurasić, Some Diophantine Triples and Quadruples for Quadratic Polynomials, J. Comb. Number Theory, 3 (2011), 123-141. 1, 2.8

[8] P. Fermat, Observations sur Diophante, Oeuvres de Fermat, Paris, (1891). 1

[9] A. Fillipin, Non-extend ability of D(-1) triples of the form $\{1,10, c\}$, Int. J. Math. Math. Sci., 35 (2005), 2217-2226. 1

[10] Y. Fujita, The D(1)-extensions of D(-1)-triples $\{1,2, \mathrm{c}\}$ and integer points on the attached elliptic curves, Acta Arith., 128 (2007), 349-375. 1

[11] L. Goldmakher, Legendre, Jacobi and Kronecker Symbols Section, Number Theory Lecture Notes, 4 pages. 1, 2.1, 2.2, $2.3,2.5$

[12] M. A. Gopalan, V. Sangeetha, M. Somnath, Construction of the Diophantine Triple involving polygonal numbers, Sch. J. Eng. Tech., 2 (2014), 19-22. 1

[13] M. A. Gopalan, G. Srividhya, Two special Diophantine triples, Diophantus J. Math., 1 (2012), $23-27$.

[14] M. A. Gopalan, S. Vidhyalakshmi, S. Mallika, Some special non-extendable Diophantine triples, Sch. J. Eng. Tech., 2 (2014), 159-160. 1

[15] M. A. Gopalan, S. Vidhyalaksfmi, Ö. Özer, A Collection of Pellian Equation (Solutions and Properties), Akinik Publications, New Delhi, (2018). 1

[16] K. S. Kedlaya, Solving constrained Pell equations, Math. Comp., 67 (1998), 833-842. 1

[17] P. Kurur (Instructor), R. Saptharishi (Scribe), Computational Number Theory, Lecture Notes, Quadratic Reciprocity (contd.) Section, 3 pages. 1, 2.6, 2.7

[18] R. A. Mollin, Fundamental Number theory with Applications, Chapman \& Hall/CRC, Boca Raton, (2008). 1

[19] Ö. Özer, A Note On The Particular Sets With Size Three, Boundary Field Prob. Comput. Simul. J., 55 (2016), 56-59. 1

[20] Ö. Özer, On The Some Particular Sets, Kirklareli Univer. J. Eng. Sci., 2 (2016), 99-108.

[21] Ö. Özer, Some Properties of The Certain Pt Sets, Int. J. Algebra Stat., 6 (2017), 117-130.

[22] Ö. Özer, On The Some Non Extandable Regular $P_{-2}$ Sets, Malaysian J. Math. Sci., 12 (2018), 255-266. 1

[23] J. Roberts, Lure of the Integers, Mathematical Association of America, Washington, DC, (1992). 1 